\title{
Optimal control algorithm of safe Island defense model in distribution network with distributed generation
}

\author{
Qinggang Zhao \\ AVIC APC Integration Equipment CO., LTD, Beijing,102206, China \\ zqg_g@163.com
}

Keywords: Distributed generation; Safe Island defense model; Continuous shortest path algorithm

\begin{abstract}
With the development of technology and people's awareness of environmental protection, more and more clean energy for power generation, such as wind power, photovoltaic power generation and so on. This type of energy is renewable and pollution-free, but it is intermittent, reliability and stability is poor. And distributed generation will form isolated island when the fault occurs in the entire power grid can make up for the shortcomings of these intermittent energy. This paper introduces the determination method of the isolated island range with only one DG, and the range of each DG island is calculated by using the continuous shortest path algorithm for multiple DG in power grid. The purpose is to ensure that the load power supply and to achieve the lowest cost at the same time.
\end{abstract}

\section{Background}

In recent years, with the increasingly stringent requirements of environmental protection, more and more clean energy such as wind power, photovoltaic power, biomass power is used in power generation. A large number of distributed generation(DG) power generation by these energy sources are incorporated into the distribution network, has brought great changes to the traditional distribution network. When the distribution network is in trouble, but the DG is still in the power generation and connected to the local load run independently is called the islanding effect ${ }^{[1]}$. Safe island defense technology refers to the use of the island effect to ensure power supply security of the important load in the range of the DG power supply, so as to protect people's lives and property safety. The key to this technology is the division of the DG power supply range, because it will reduce the reliability of the power supply if the responsible scope is too large and will waste energy if too small. In order to solve this problem, we provide a range partitioning method with single DG and multiple DG in this paper.

\section{The objective function and constraint conditions of DG island division}

There are a lot of different levels of load in the power grid, we will be graded according to their important level first. In the formation of an isolated island, should be included the high level of importance load as much as possible. So the target function of island division is as follows:

Among them, $n$ represents the number of isolated islands; $\mathrm{H}_{\mathrm{i}}$ represents a collection of all the loads in the $i$ Island; $L_{j}$ is the type of load element within the $H_{i} ; \lambda_{L j}$ is the weight of load type $L_{j} ; P_{L j}$ is the power size of the load type. In this paper, we divide the load level into 1,2, and 3 and their weights are 1,2,3, respectively. The smaller the weights represent the load more important.

As the island's capacity is limited, so it is also required to meet the following constraints:

(1) Power balance in isolated island. This constraint conditions require that the generation of DG in the isolated island should be balanced with the load.

(2) Current constraint. The current on each line should not exceed the limit value after the isolated island formation.

(3) Voltage constraint. The voltage level of each load should be maintained within the scope of the requirements to ensure the quality of power supply after the island formation.

(4) Topological constraint. In this constraint, need a connecting path between the load node and 
the DG in the island, and the island is in a radial run.

\section{Determination of the island range}

Due to the limited capacity of DG, so the scope of the island to provide power when the grid is in trouble is also limited. Determine the maximum range of the island according to the capacity of the DG and remove excess load, this can effectively improve the computational efficiency.

We will use an example to demonstrate the process of determining the island range. The capacity of the DG is $800 \mathrm{KW}$, the load in the area is shown in Table land containing region show in figure 1 .

Table1 The load of DG within the connected region

\begin{tabular}{|c|c|c|c|c|c|c|c|c|c|c|c|c|c|}
\hline Load number & 1 & 2 & 3 & 4 & 5 & 6 & 7 & 8 & 9 & 10 & 11 & 12 & 13 \\
\hline Load & 8 & 31 & 23 & 45 & 27 & 19 & 48 & 30 & 38 & 17 & 36 & 24 & 34 \\
\hline capacity/KW & 0 & 0 & 0 & 0 & 0 & 0 & 0 & 0 & 0 & 0 & 0 & 0 & 0 \\
\hline
\end{tabular}

Figure 1 Connected region containing DG

As shown in Figure 1 with the DG connected region, the load parameters of DG's power supply path are shown in Table 2.

Table 2 The load parameters of DG's power supply path

\begin{tabular}{ccc}
\hline $\begin{array}{c}\text { Number of power supply } \\
\text { path }\end{array}$ & $\begin{array}{c}\text { Load number on power } \\
\text { supply path }\end{array}$ & Total load/KW \\
\hline 1 & $1,4,9,13$ & 1250 \\
2 & $2,5,10$ & 750 \\
3 & 2,6 & 500 \\
4 & $3,7,11$ & 1070 \\
5 & $3,8,12$ & 770 \\
\hline
\end{tabular}

As shown in Table 2, the power supply path of DG 1 and 4 contains more than DG of the total load capacity. So it is needed to remove the lowest layer of the load on the two paths until the load on the path is in the range of DG capacity. After eliminating the nodes 13 ,9and 11, the total load of path 1 and path 4 changed to $530 \mathrm{~kW}$ and $710 \mathrm{~kW}$ respectively. At this point, load on all paths are in the range of DG capacity. The maximum power supply range of the DG can be obtained, as shown in figure 2.

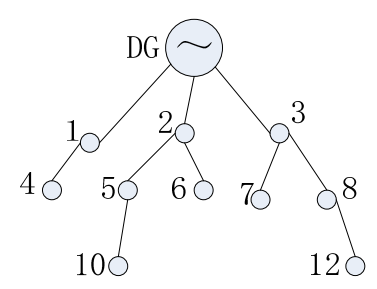

Figure 2 Maximum power supply range of DG

The above is the fault area containing only one DG, if the fault area contains more than one DG and some loads are in the range of multiple DG power supply at the same time. At this time we need to consider the cost of power generation DG, the share load will be included into the lower cost DG. Specific methods will be introduced in the following.

\section{Continuous shortest path algorithm}

The essence of DG Island partitioning problem is under the condition of DG capacity constraint, 
in order to find the power supply path of DG for more important load. This problem can be understood as the minimum cost maximum flow problem in graph theory, continuous shortest path algorithm is a fast and effective algorithm to solve the minimum cost maximum flow problem ${ }^{[2]}$. For a more detailed description of the shortest path algorithm, connected region with multiple DG in the XX city present in figure 3 as an example. There are one school, one factory, one hospital and two DG in this city.

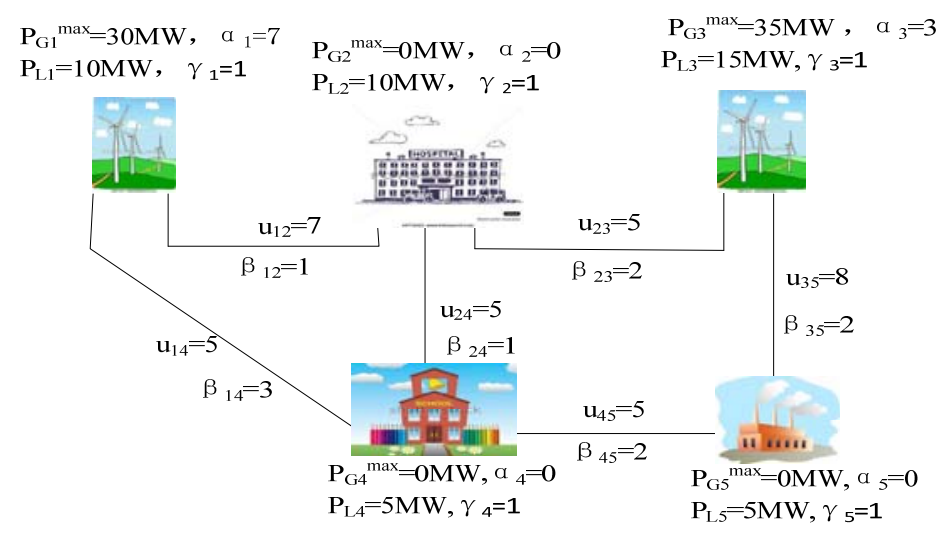

Figure 3 Connected region with multiple DG

As shown in Figure $3, \mathrm{P}_{\mathrm{G}}{ }^{\max }$ is the upper limit of active capacity of DG, $\mathrm{P}_{\mathrm{L}}$ is the load active power, $\alpha, \beta, \gamma$ is power generation cost, line transmission cost, load cost for DG respectively. Continuous shortest path algorithm can not be directly applied to the distribution network, it is required to transform the system shown in Figure 3 into a graph theory model. The graph theoretic model of the system is shown below:

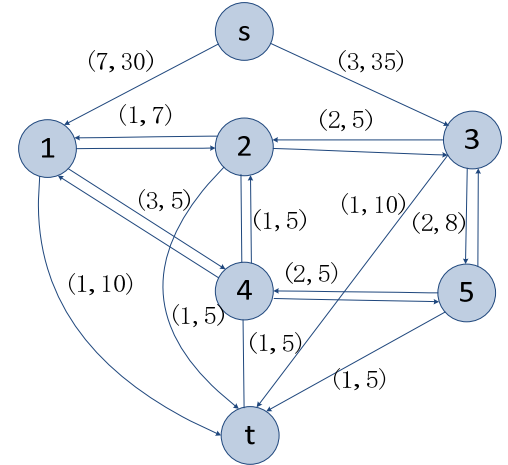

Figure 4 Graph theoretic model of connected regions with multiple DG

In Figure 4, $s$ is the virtual source point, $t$ is the virtual sink point, this is done in order to change the power supply into a single power supply, multi sink point into a single sink point. Figures in brackets refer to the transport cost and the remaining capacity of the branch.

The core of the shortest path is to update the distance label of each node $\pi_{i}$, its specific meaning can be understood as the source point $s$ to the node i shortest path length, each vertex can be linked by this setting. Continuous shortest path algorithm is to continuously update the distance of each node label until all nodes are the shortest path to meet the optimal conditions of the shortest path:

In the label update process, when $\pi_{j}>\pi_{i}-\beta$ ij , let $\pi_{j}=\pi_{i}-\beta$ ij.

Before the start of the shortest path, in order to obtain the shortest path from source point $s$ to sink point $t$, Define the following three arrays:

(1) $\mathrm{S}[\mathrm{n}]: \mathrm{S}[\mathrm{i}]$ indicates whether the node $\mathrm{i}$ is traversed when searching for the shortest path, 1 means yes and 0 means no.

(2)label[n]: label[i] represents the length of the shortest path from the source point $\mathrm{s}$ to the node $\mathrm{i}$. Its value is equal to $\pi_{\mathrm{i}}$.

(3)path[n]: path[i] represents the number of the previous node of the node $\mathrm{i}$ On the shortest path from the source point $\mathrm{s}$ to the node $\mathrm{i}$.

At the time of operation, the first step is to initialize the data; The second step is to update the source point s related nodes in the array of parameters; And then choose to meet the requirements of 
the label[i], to update path[i] and $\mathrm{S}[\mathrm{i}]$; If $\mathrm{S}[\mathrm{i}]=1$, reverse tracking path array, Determine the shortest path, if it is not then return to the second step to re cycle.

Sequential shortest path algorithm to determine the shortest path of a source point $\mathrm{s}$ to point $\mathrm{t}$ calculation process and to make the path to meet the constraints of the parameter setting process ${ }^{[3]}$, As shown in Figure 5 to determine the path of the system after the s-3-t graph theory model.

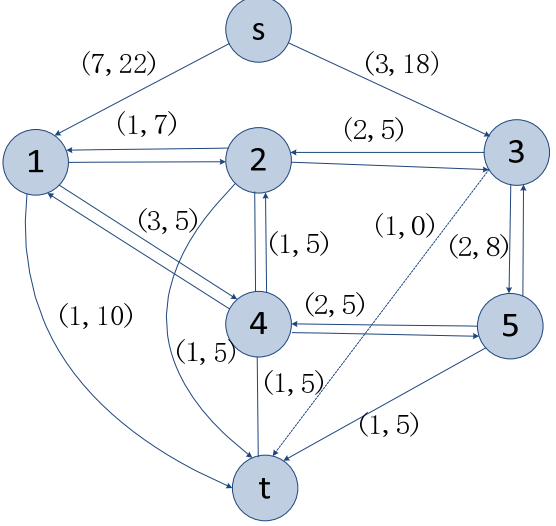

Figure 5 System graph theory model for determining path s-3-t

Algorithm to determine the shortest path after the update of the parameters, the initial array s, label, path, and then re start looking for a $\mathrm{S}$ to $\mathrm{t}$ the shortest path. Continuous shortest path is through the $s$ to find the shortest path to $t$, Until the DG available power generation is less than the minimum system load $\mathrm{L}_{\min }$ or restore the power supply load is equal to the total system load $\mathrm{L}_{\text {sum. }}$.

The flow chart of DG island partitioning strategy based on the shortest path algorithm is shown in Figure 6.

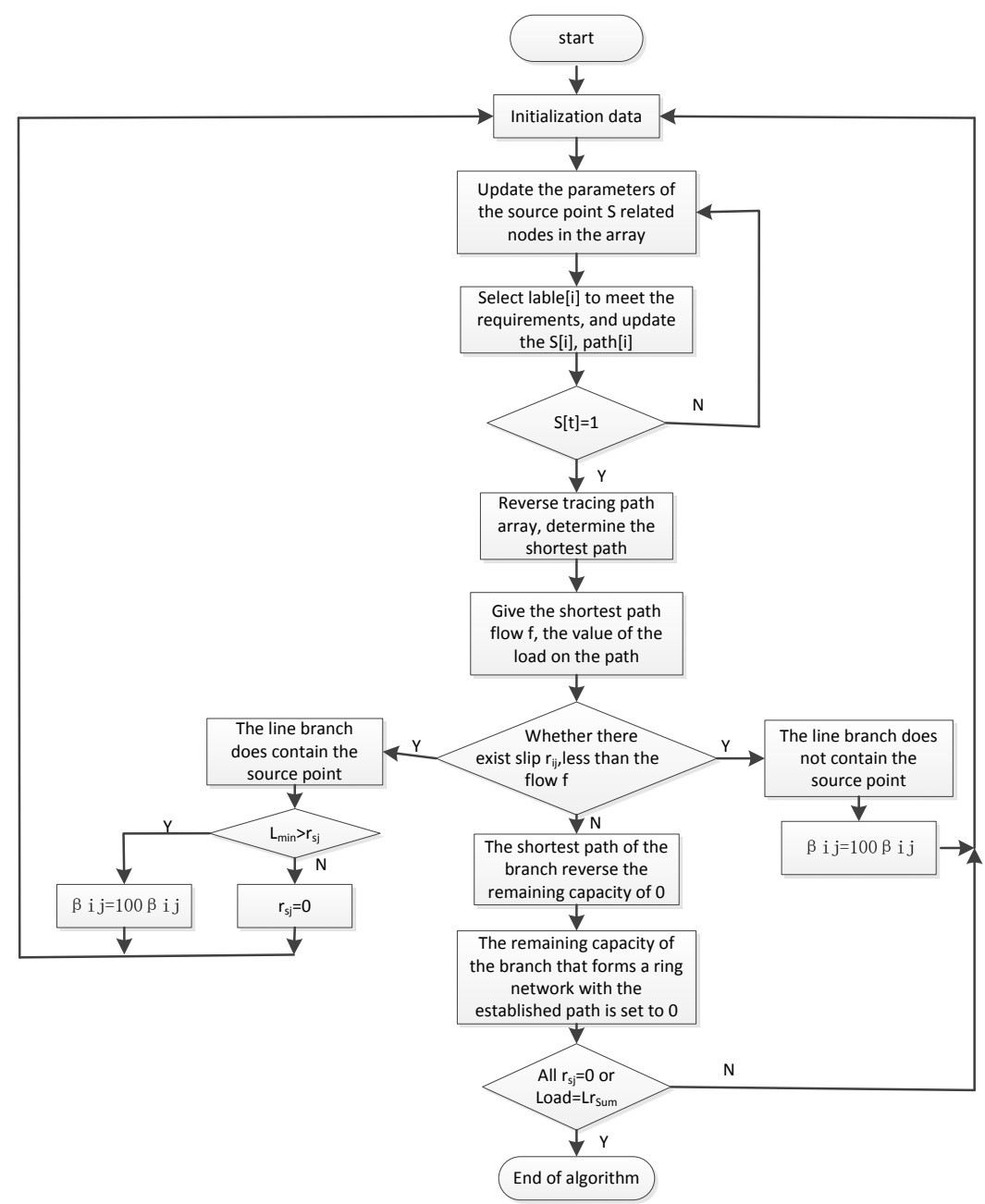

Figure 6 DG island partitioning strategy based on the shortest path algorithm 


\section{Conclusion}

With the development of technology and people's awareness of environmental protection, there must be more and more DG into the power supply network in the future. Through the optimization of the control algorithm of the safe island defense model in the distribution network with distributed generation, can greatly improve the reliability and stability of this kind of distribution network power supply. By scientific DG Island division, can be in the case of the minimum cost to ensure that as much as possible without power outage, it is a great significance to protect the people's lives and property.

\section{Reference}

[1] Khamis A, Shareef H, Mohamed A, et al. Islanding detection in a distributed generation integrated power system using phase space technique and probabilistic neural network[J]. Neurocomputing, 2015, 148:587-599.

[2] Hu L, Peng B, Jiang Y, et al. Model and Algorithm for Continuous Time-Varying Shortest Path Problem[C]// ICTE 2011. ASCE, 2015:271-276.

[3] El-Sherbeny N A. The Algorithm of the Time-Dependent Shortest Path Problem with Time Windows[J]. Applied Mathematics, 2014, 05(17):2764-2770.

[4] Hirodontis S, Li H, Crossley P. Load shedding in a distribution network[C]//Sustainable Power Generation and Supply, 2009. SUPERGEN'09. International Conference on. IEEE, 2009: 1-6.

[5]Costache M, Tudor V, Almgren M, et al. Remote control of smart meters: friend or foe?[C]//Computer Network Defense (EC2ND), 2011 Seventh European Conference on. IEEE, 2011: 49-56.

[6]Islam M R, Gabbar H. Analysis of Microgrid protection strategies[C]//Smart Grid Engineering (SGE), 2012 IEEE International Conference on. IEEE, 2012: 1-6. 